\title{
Efficacy of hydraulic pressure in transcrestal sinus membrane elevation followed by immediate implant placement without bone graft
}

Original Article
Salah ElAbbasy

Department of Oral and Maxillofacial Surgery, Faculty of Oral and Dental Medicine, Cairo University, Cairo, Egypt

\begin{abstract}
Aim: To evaluate the efficacy of hydraulic pressure in transcrestal sinus membrane elevation followed by immediate implant placement without bone graft.

Materials and Methods: A total number of 10 implants were inserted in eight patients selected from the outpatient clinic of Oral and Maxillofacial Surgery Department, Faculty ofDentistry, Cairo University.Drilling of the alveolar ridge was carried out using a 2.0 pilot drill to a depth of $1 \mathrm{~mm}$ away from the sinus floor. Osteotomes were used sequentially to condense and widen the osteotomy site until the desired diameter of the implant is reached. The remaining $1 \mathrm{~mm}$ of bone was separated from the floor of the sinus using an osteotome followed by insertion of The ZimmerSinus Lift Balloonto gentlyelevate the sinus membrane.

Results: No post-operative complications were recorded in terms of sinus perforation, infection, nasal bleeding or wound dehiscence during the follow up period.The changes in bone density from immediate postoperative to 6 months postoperative showed a significant increase in bone density in the mesial and distal sides of the implants inserted. All the implants inserted were successfully osseointegrated. The mean time for the surgical procedure was found to be $14.6 \pm 1.9$ minutes.

Conclusion: Transcrestal balloon sinus lifting is minimally invasive, safe and reliable. It results in reduced postoperative pain and complications when compared to other techniques. No bone graft is necessary to be placed. This technique proved to be a time and money saving procedure.
\end{abstract}

Key Words: Balloon lift technique, simultaneous implant placement, transcrestal sinus elevation.

Received: 28 May 2019, Accepted: 30 May 2019

Corresponding Author: Salah ElAbbasy, Department of Oral and Maxillofacial Surgery, Faculty of Oral and Dental Medicine, Cairo University, Tel.: 01001408066, E-mail: drsalahosman@hotmail.com

ISSN: 2090-097X, January 2019, Vol. 10, No. 1

\section{INTRODUCTION}

The posterior maxilla usually lacks sufficient bone height for placement of dental implantsmainly due to pnematization of the maxillary sinus. Many surgical techniques have been introduced to overcome this problem including Le Fort I maxillary down fracturing, implant tilting, onlay bone grafts and sinus elevation procedures $^{[1,2,3,4,5]}$.

With sinus lifting procedures, surgeons use twoapproaches to elevate the sinus membrane: the lateral window approach and the transcrestal approach ${ }^{[5]}$.

In the lateral window approach, the sinus membrane is accessed and elevated through a window made in the buccal wall of the maxillary sinus where implants can be inserted immediately or in another stage depending on the height of the ridge which determines the ability to obtain primary implant stability ${ }^{[6]}$. Although the lateral window technique is associated with high success rate for the the implants placed, it suffers from many shortcomings including sinus perforation, bleeding, infection andinfraorbital nerve lacerations, this technique is time consuming and requires high surgical skills ${ }^{[7,8]}$.

Transcrestal approach using osteotomes is a simple techniquewhere the dental implants are placed simultaniously with the elevation procedure. This approach results in bone condensation and better implant stability together with reduced patient discomfort and morbidity ${ }^{[9]}$, however the main limitiation for this technique is allowing membrane elevation of only $3 \pm 0.8 \mathrm{~mm}^{[10,11]}$.

Modification of transcrestal approach was introduced in 2003 where hydraulic pressure via a balloon was applied to elevate the sinus membrane with a minimal risk of perforation and with an advantage of elevating the sinus membrane of up to $15 \mathrm{~mm}$ so it can be used when the residual bone height is $3 \mathrm{~mm}$ in contrast to the conventional 
osteotome technique which can't be used if the residual bone height is less than $5 \mathrm{~mm}^{[12]}$.

\section{MATERIALS AND METHODS}

A total number of 10 implants were inserted in eight patients selected from the outpatient clinic of Oral and Maxillofacial Surgery Department, Faculty of Dentistry, Cairo University. All the patients signed a written consent of approval to participate in our study.

All the patients selected were free from any local or systemic disease that contraindicate the sinus lifting surgery or may complicate the healing process. Any patients with maxillary sinus disease, former sinus surgery like Caldwell luc operations, severe sinus floor convolutions, extremely narrow sinuses and unfavorable inter maxillary relationship were excluded from our study.

Preoperative cone beam radiographs were performed to examine the sinus and to determine the residual maxillary alveolar ridge height. Patients with residual alveolar bone height of 3-7 mm were included in our study, however, those with bone height of less than $3 \mathrm{~mm}$ were excluded from the study as primary implant stability can't be achieved.

All the patients received strict oral hygiene measures with $0.12 \%$ chlorohexidinegluconate 3 times daily for one week prior to the surgery.

Buccal and palatal infiltration anaesthesia of $4 \%$ Articaine hydrochloride with epinephrine 1:100,000was injected for all the patients. A full thickness crestal incision slightly palatal to the ridge was carried out over the proposed implant site. A full thickness mucoperiosteal flap was elevated and the underlying bone of the ridge was exposed.

Drilling of the alveolar ridge was carried out using a $2.0 \mathrm{~mm}$ pilot drill to a depth of $1 \mathrm{~mm}$ away from the sinus floor. Osteotomes were used sequentially to condense and widen the osteotomy site until the desired diameter of the implant was reached. The remaining $1 \mathrm{~mm}$ of bone was separated from the floor of the sinus using an osteotome with gentle tapping (Fig. 1).

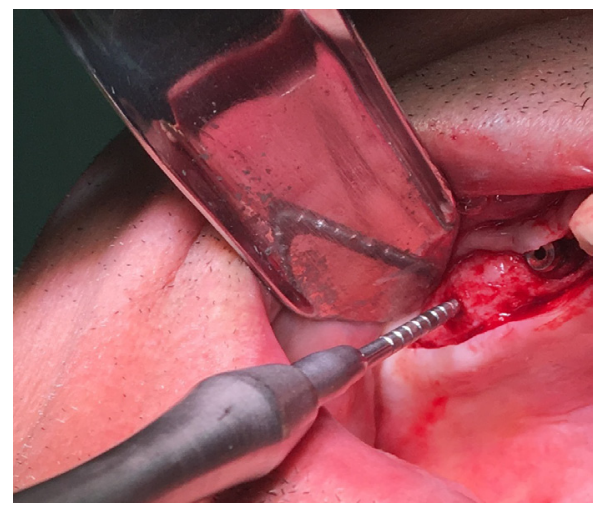

Fig. 1: Showing the osteotome used to separate the remaining 1 $\mathrm{mm}$ of bone from the floor of the sinus
The Zimmer Sinus Lift Balloon (Fig. 2) was used to gentlyelevate the sinus membrane. It isa pneumatic device consisting of a $5 \mathrm{ml}$ syringe, polyvinylchloride tubing, and a metal shaft with a tip connectedto a latex mini balloon with an inflation capacity ofapproximately $5 \mathrm{cc}$. Each $1 \mathrm{cc}$ of saline solution injected into the balloon results in $6 \mathrm{~mm}$ of membrane elevation.

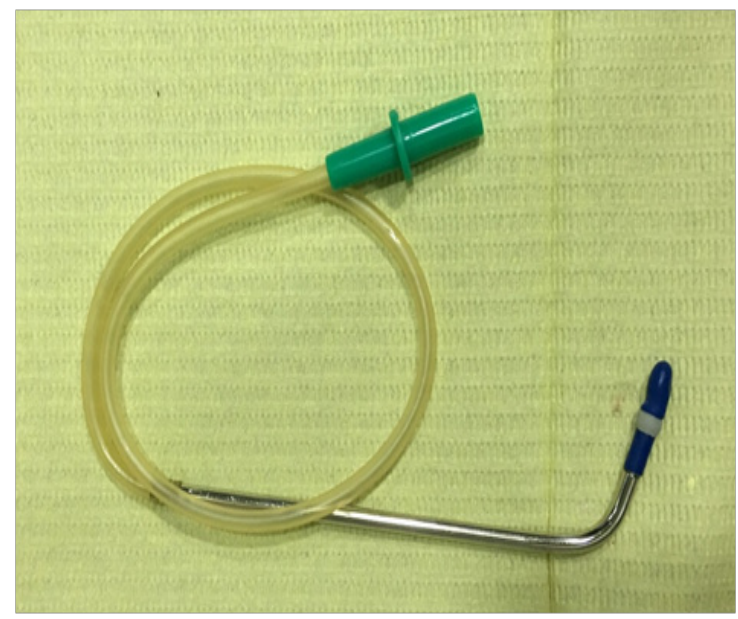

Fig. 2: Showing Zimmer sinus lift Balloon

Prior to balloon insertion through the osteotomy, its integrity must be checked by inflation and deflation for several times.

The balloon was inserted into the osteotomy until it reaches the subantral space and saline was injected inside the balloon through a plastic syringe (Fig. 3).Then the balloon was deflated and carefully removed from the sinus.

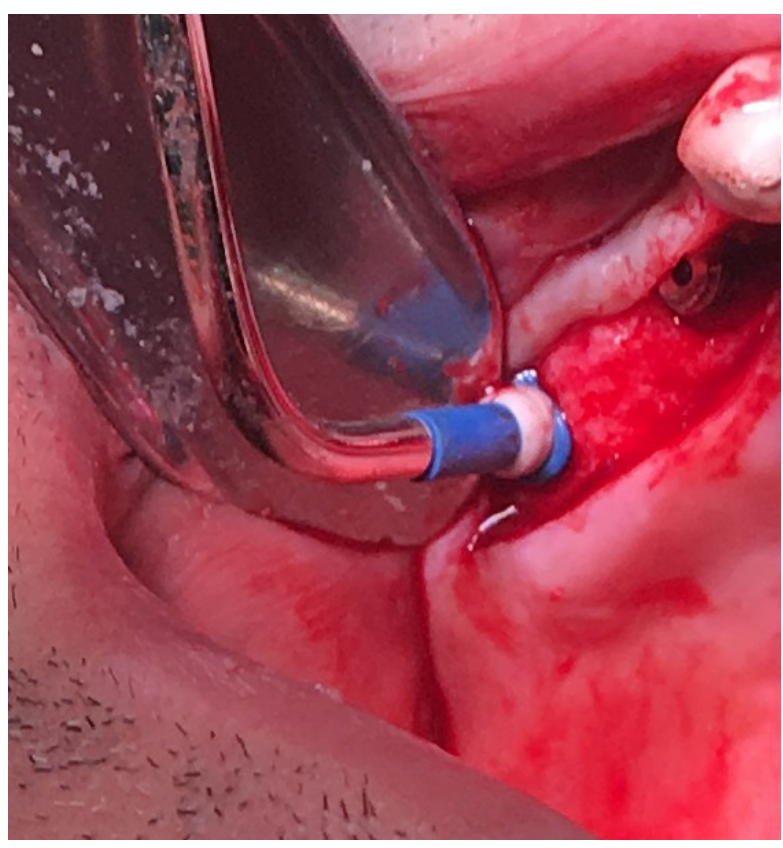

Fig. 3 : Showing sinus membrane elevation using the balloon 
The sinus membrane was examined for any tear or perforation by asking thepatient to blow gently through the nose with the nostrils pinched and checking for a mist on a mirror placed below the osteotomy site.

Implant of selected height and diameter was inserted into the osteotomy site without bone grafting where the sinus membrane will be tented over the implant apex and the flap was returned in place and sutured with $3 / 0$ black silk sutures.

The patients were instructed to avoid any actions that might result in high intranasal pressure like sneezing, nose blowing and drinking with straws for 1 week postoperatively.

Postoperative prescription of antibiotic Clindamycin $\mathrm{HCl} 300 \mathrm{mg}$ tablets every 8 hours for 5 days, Ketoprofen 50 $\mathrm{mg}$ analgesic tablets given three times daily for 3-5 days, Decongestant nasal drops three times daily for 10 days and Mouth rinsing with $0.12 \%$ Chlorohexidinegluconate 3 times per day for 1 week.

\section{Follow up examination:}

1-Clinical evaluation was performed next day and after 7 days postoperatively including:

-Pain using the Visual Analogue Scale of pain (VAS)

-Edemaevaluated by visual descriptor scale 13

-Complications in terms ofinfection, nasal bleeding and wound dehiscence

2-Radiographic evaluation was performed for each patient by obtaining digital panoramic radiographs [Orthotomograph OT100, Instrumentarium Imaging, GE corporation, Finland ] loaded with the cassette and the exposure factors were set for an adult patient.The radiographs were performed immediate postoperatively and after 6 months postoperatively.

\section{Image analysis:}

Peri-implant bone density changes were analyzed

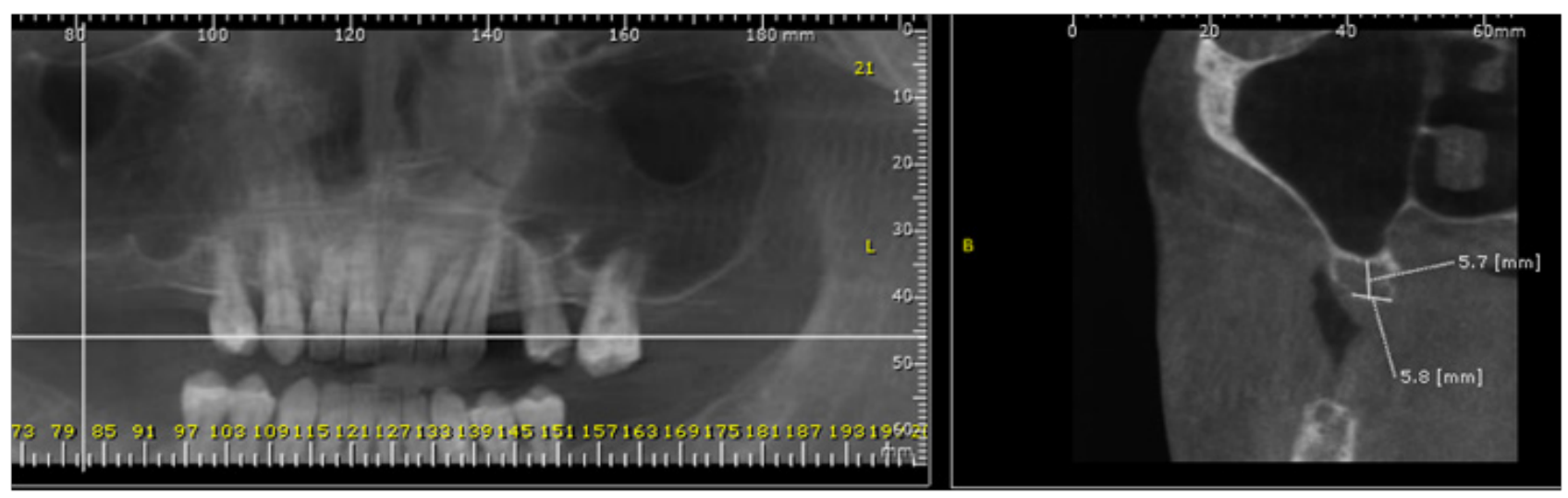

Fig. 5: Showing preoperative cone beam with residual ridge height of $5.7 \mathrm{~mm}$ to evaluate the bone density around the implants. The software of the digital panorama was used to calculate the mean gray values at the mesial and distal aspects of each implant by drawing three lines parallel to each other and $1 \mathrm{~mm}$ apart from each other, where the first line was drawn tangential to the threads of the implant. The mean grey value along each line was determined and the total mean bone density along the three lines was calculated and included in the statistical analysis (Fig. 4).

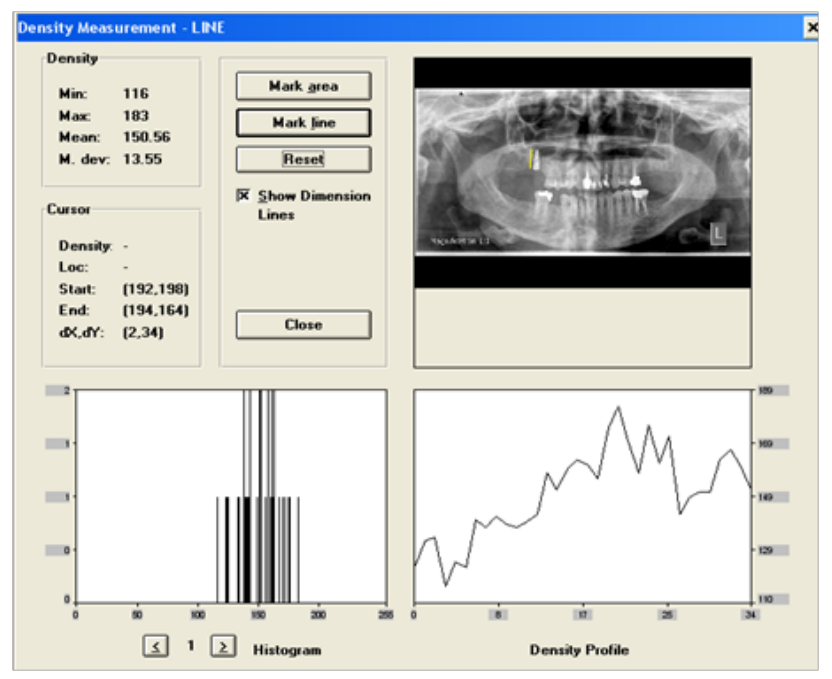

Fig. 4: Showing the measurement of bone density at the distal side of the implant immediate postoperatively

\section{RESULTS}

In our study, ten cases of sinus floor elevation were performed on eight patients ( 5 males and 3 females) with an age range of 34-59 years old.

The preoperative mean height of the residual alveolar ridge was $5.63 \pm 1.82 \mathrm{~mm}$ with a range of 3.5-6.9 mm (Fig. 5). 


\section{Clinical Results:}

No cases of sinus perforation was observed where no mist appeared on the mirror placed below the osteotomy site for all the patients.

Pain: Six patients experienced mild pain with VAS range from 2-4, while two patients experienced moderate pain with VAS range 5-7 in the next day after the surgical procedure. However, at day 7 , none of the patients presented any kind of pain.

Edema: Only two patients suffered from mild edema next day after surgery which was completely resolved after 7 days post-surgical.

Post-operative complications: No post-operative complications were recorded in terms of infection, nasal bleeding or wound dehiscence duringthe follow up period.

\section{Radiographic results:}

All the panoramic x-rays revealed deposition of bone on the mesial and distal aspects of the dental implants after 6 months postoperatively (Fig. 6).

The mean and standard deviation(SD) for the bone density of mesial and distal sides of the inserted dental implants was recorded immediate post operatively and after 6 months postoperatively (Table 1).

Comparing the bone density between the mesial and distal sides of the implants, there was no statistically significant difference between the 2 sides immediate postoperative and after 6 months post-operative (Table 2).

The changes in bone density from immediate postoperatively to 6 months postoperatively showed a significant increase in bone density in the mesial and distal sides (Table 3).

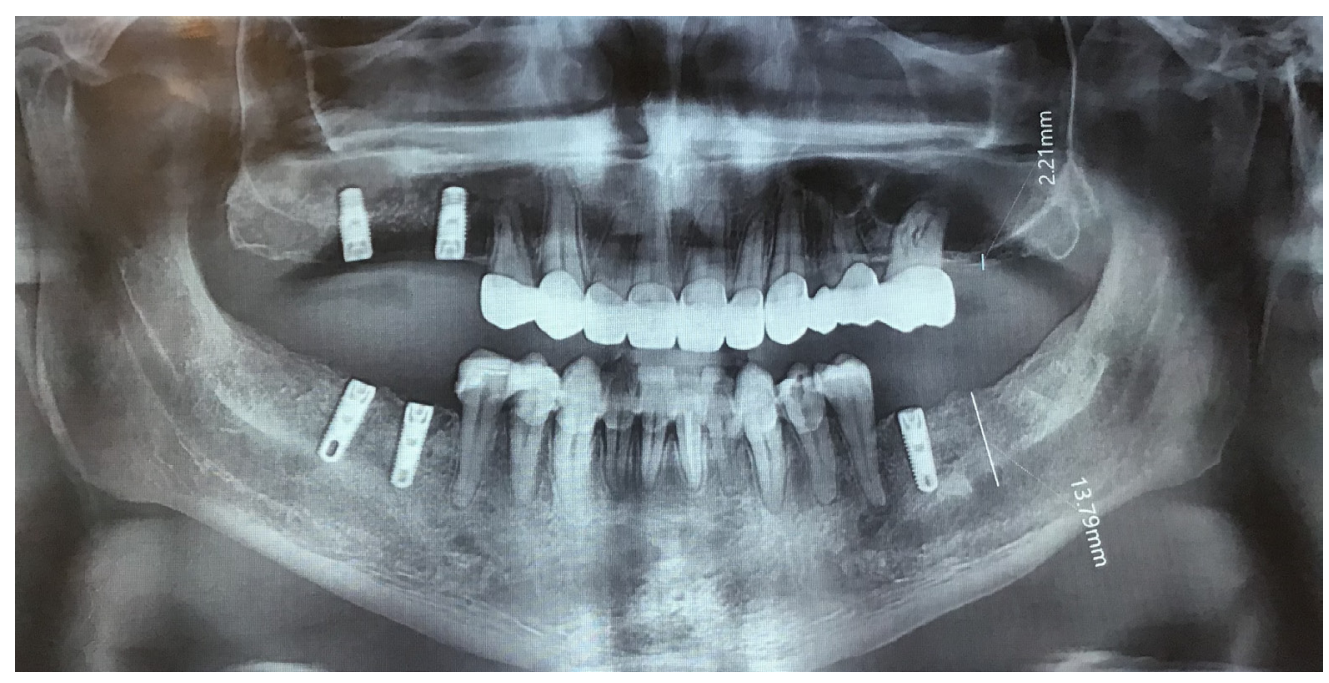

Fig. 6: Six months postoperative panoramic x-ray showing mesial and distal bone formation around 2 implants placed in posterior right maxillary area

Table 1: Showing the mean bone density on mesial and distal aspects of the implants

\begin{tabular}{|c|c|c|c|c|}
\hline & \multicolumn{2}{|c|}{ Immediate post-operative } & \multicolumn{2}{|c|}{6 months postoperative } \\
\hline & Mesial & Distal & Mesial & Distal \\
\hline Mean & 111.2 & 120.6 & 139.8 & 145.4 \\
\hline SD & 16.2 & 17.5 & 12.8 & 13 \\
\hline
\end{tabular}


Table 2: Showing the mean, standard deviation (SD) values and results of paired t-test for the comparison between mesial and distal sides

\begin{tabular}{|c|c|c|c|c|c|}
\hline & \multicolumn{2}{|c|}{ Mesial } & \multicolumn{2}{|c|}{ Distal } & \multirow[b]{2}{*}{$P$-value } \\
\hline & Mean & $\mathrm{SD}$ & Mean & $\mathrm{SD}$ & \\
\hline Immediate & 111.2 & 16.2 & 120.6 & 17.5 & 0.136 \\
\hline 6 Months & 139.8 & 12.8 & 145.4 & 13 & 0.27 \\
\hline
\end{tabular}

Table 3: Showing the means, standard deviation (SD) values and results of paired t-test for studying the changes in bone density

\begin{tabular}{|c|c|c|c|c|}
\hline & & Mean difference & SD & $P$-value \\
\hline Mesial & Immediate- 6 months & 28.6 & 14.5 & $<0.001 *$ \\
\hline Distal & Immediate- 6 months & 24.8 & 15 & $<0.001 *$ \\
\hline
\end{tabular}

The distance from the floor of the sinuses till the apex of the implants wasmeasured to evaluate the amount of sinus elevation gained. The mean sinus elevation gained was $6.9 \mathrm{~mm}$ where the minimal elevation gained was 5.1 $\mathrm{mm}$ and the maximum was $8.2 \mathrm{~mm}$

All the implants inserted in this study were successfully osseointegrated without any signs of implants failure.

\section{III-Time required for the surgical procedure:}

The time for the surgical procedure was recorded starting from the incision until the last suture was placed. The mean time for the surgical procedure was found to be $14.6 \pm 1.9$ minutes

\section{DISCUSSION}

In the present study, transcrestal balloon elevation proved to be a simple, safe and reliable technique with no postoperative complications. This in contrast to the findings of Kfir et al. ${ }^{[14]}$ and Zimbler et al. ${ }^{[15]}$ who reported major complications associated with the traditional techniques such as membrane perforations, bleeding and infections.

In our study, implants were placed simultaneously with the sinus lifting where the minimal residual alveolar ridge height recorded was $3.5 \mathrm{~mm}$. All the patients with residual bone height less than $3 \mathrm{~mm}$ were excluded from the study as this bone height is insuffiecient to provide primary implant stability. This is in agreement with the study of Fenner et al. ${ }^{[16]}$ who recommended a two stage surgical protocol of bone grafting and delayed implant insertion if the residual bone height is less than $3 \mathrm{~mm}$.

In this study, tenting the sinus membrane over the dental implants without bone graft maintains a space between the floor of the sinus and the membrane. This allows blood clot to be formed followed by resorption and deposition of bone cells. This could explain the appearance of new bone around the dental implants and the significant incresase of bone density results around the implants after 6 months. This is consistent with the study performed by Linde et $a l^{[17]}$ who reported that bone regeneration will occur after creation and maintenance of an isolated space between the periosteum and the calvarial cortex after sinus floor elevation. It is conceivable that formation of new bone in the maxillary sinus does not require the presence of various grafts as scaffolds.

It is of concern if placement of graft materials could result in stimulation of new bone formation, an explanation that met with the findings of Tong et al..$^{[18]}$ and Fabbro et al. ${ }^{[19]}$ who reported that current allograft materials, which are reportedly bio-inert, osteoconductive, or questionably osteoinductive, are not expected to stimulate new bone formation.

Transcrestal balloon sinus lifting is a time saving procedure where the mean time for our surgeries was found to be 14.6 minutes. This result is in agreement with the findings of Yukinobu et al. ${ }^{[20]}$ who reported that a typical single tooth or multi tooth procedure that generally takes 30 mins will take only 10-15 minutes with the 
balloon lift technique. In addition, Eggers et al. ${ }^{[21]}$ and Leclercq et $a .^{[22]}$ reported that sinus elevation using ultrasonic instruments is time consuming together with its poor efficiency.

The mean sinus elevation gained in our study was $6.9 \mathrm{~mm}$. This technique overcomes the limitation of the conventional osteotome technique which provide less gain in sinus elevation. This coincides with the findings of Krafft et al ${ }^{[9]}$, Tatum ${ }^{[10]}$ and Summers ${ }^{[11]}$ who reported that osteotome techniques results in bone condensation and better implant stability together with reduced patient discomfort and morbidity, however, the main limitiation for this technique is allowing membrane elevation of only $3 \pm 0.8 \mathrm{~mm}$.

\section{CONCLUSION}

Transcrestal balloon sinus lifting is minimally invasive, safe and reliable. It results in reduced postoperative pain and complications when compared to other techniques. No bone graft is necessary to be placed. This technique proved to be time and money saving procedure.

\section{CONFLICT OF INTEREST}

There are no conflicts of interest.

\section{REFERENCES}

1. Sailer HF: A new method of inserting endosseous implants in totally atrophic maxillae. J CraniomaxillofacSurg 17:299,1989.

2. Adell R, Lekholm U, Grondahl $\mathrm{K}$, et al: Reconstruction of fixtures in immediate autogenous bone grafts. Int $\mathrm{J}$ Oral Maxillofac Implants 5:233, 1990.

3. Isaksson S, Alberius P: Maxillary alveolar ridge augmentation with onlay bone grafts and immediate endosseous implants. J Craniomaxillofac Surg 20:2,1992.

4. Boyne PJ, James RA: Grafting of the maxillary sinus floor with autogenous marrow and bone. $\mathrm{J}$ Oral Surg 38:613, 1980.

5. Richter HE, Boyne PJ: New concepts in facial bone healing and grafting procedures. J Oral Surg 27:557, 1969.

6. Pjetursson, B.E., Tan, W.C., Zwahlen, M. and Lang,N.P.A systematic review of the success ofsinus floor elevation and survival of implantsinserted in combination with sinus floor elevation. Journal of Clinical Periodontology 35: 216-240, 2008.

7. Misch CE. Maxillary sinus augmentation for endosteal implants: organized alternative treatment plans. Int J Oral Implantol; 4:49-58,1987.

8. Viña-Almunia J, Peñarrocha-Diago $\mathrm{M}$, Pen arrocha-Diago M. Influence of perforation of the sinus membrane on the survival rate of implants placed after direct sinus lift. Literature update. Med Oral Patol Oral Cir Bucal14:E133-6, 2009.

9. Krafft, T., Graef, F., Winter, W., Wichmann, M. \&Karl, M. Use of osteotomes for implant bedpreparation - effect on material properties of boneand primary implant stability. Journal of Oral Implantology39(s1):241-247,2013.

10. Tatum, H. Jr. Maxillary and sinus implantreconstructions. Dental Clinics of North America30: 207-229, 1986.

11. Summers, R.B. A new concept in maxillaryimplant surgery: the osteotome technique. Compendium 15: 152, 154-156, 158 passim; quiz 162, 1994.

12. Muronoi, M.,Xu, H., Shimizu, Y. \&Ooya, K. Simplified procedure for augmentation ofthe sinus floor using a haemostatic nasal balloon. British Journal of Oral and Maxillofacial Surgery 41: 120-121, 2003.

13. Yokobe J, Kitahara M, Matsushima M, Uezono S. Preference for different anchor descriptors on visual analogue scales among Japanese patients with chronic pain.PLoS One.9(6):e99891, 2014.

14. Kfir E, Kfir V, Eliav E, Kaluski E: Minimally invasive antral membrane balloon elevation; report of 36 procedures. J Periodontol. 78:2032, 2007.

15. Zimbler MS, Levowitz Ra, Glickman R, Brecht LE, Jacob JB: Antral augmentation. Osseointegration and sinusitis, the autolaryngologist'sprespective. Am J Rhinol.12 :311, 1998.

16. Fenner M, Vairaktaris E, Stockmann P, Schlegel KA, Neukam FW, Nkenke E. : Influence of 
residual alveolar bone height on implant stability in the maxilla: an experimental animal study. Clin Oral Implants Res. 20(8):751, 2009.

17. Linde A, Thoren C, DahlinC : Creation of new bone by anosteopromotive membrane technique: An experimental studyin rats. J Oral Maxillofac Surg 51:892, 1993.

18. Tong DC, Rioux K, Drangsholt $\mathrm{M}$, et al: A review of survival rates for implants placed in grafted maxillary sinuses using meta-analysis. Int J Oral Maxillofac Implants. 13:175, 1998.

19. Fabbro MD, Testori T, FrancettiL : Systematic review of survival rates for implants placed in the grafted maxillary sinus. Int $\mathrm{J}$ Periodontics Restorative Dent 24:565, 2004.

20. Yukinobu F, Sennichi S, Kazuko K., Sonoko K, Yutaka D :Evaluation of subantral membrane balloon elevation technique using cone-beam $\mathrm{CT}$. J Gifu Dent. Soc. 36:100, 2009.

21. Eggers G, Klein J, Blank J, Hassfeld S. : Piezosurgery, an ultrasound device for cutting bone and its use and limitations in maxillofacial surgery. Br J Oral MaxillofacSurg42:451, 2004.

22. Leclercq P, Zenati C, Dohan DM.: Ultrasonic bone cut part 2: state-of-the-art specific clinical applications. J Oral MaxillofacSurg 66:183,2008. 